speakers shared their experiences from Egypt and the US. Discussions focused on prenatal versus postnatal, early-onset versus late-onset, and hospital versus community acquired neonatal infections. Five topics represented high priorities for research in Egypt: 1) maternal vaginal colonisation patterns and maternal vaginal screening practices for common and emerging pathogens, 2) risk factors associated with hospital-acquired infections in delivery rooms and neonatal intensive care units, 3) antimicrobial resistance among pathogens affecting newborns in intensive care units, 4) education and compliance with infection control measures among staff, and 5) presentation and risk factors for neonatal infections associated with home deliveries. Webinar conferences will be conducted with each team to mature their project. A second workshop will be organised to develop a grant proposal for each research project to be submitted to international funding agencies.

Conclusion To address neonatal infections related mortality and morbidities, stakeholders involved in the care of the newborns in Egypt need to develop a prioritised future research agenda. A central taskforce need to facilitate the assembly of multicenter, multidisciplinary teams across the country to study these issues in collaboration with international expertise and funding resources.

\section{PO-0555 PREDICTIVE VALUE OF ADMISSION SURFACE SWABS IN EARLY-ONSET NEONATAL SEPSIS IN EXTREMELY LOW BIRTH WEIGHT (ELBW) INFANTS IN A NEONATAL INTENSIVE CARE UNIT (NICU)}

${ }^{1} \mathrm{M}$ Mustapa, ${ }^{1 J}$ Egyepong, ${ }^{2}$ AK Abdul-Rahman. ${ }^{1}$ Neonatal Intensive Care, Luton and Dunstable University Hospital NHS Foundation Trust, Luton, UK; ${ }^{2}$ Public Health Directorate, Luton Borough Council, Luton, UK

\subsection{6/archdischild-2014-307384.1197}

Introduction Early Onset Neonatal Sepsis (EONS) is a major contributor to morbidity and mortality in ELBW infants. Admission surface swab cultures (SSC) form part of admission surveillance cultures, however its place in the management of EONS is questionable.

Objective To determine:

- Sensitivity, specificity and positive predictive value of SSC.

- If culture result would reflect on mean CRP value in first 72 hrs.

- If maternal swabs and mode of delivery correlated with microbiological result in the baby.

\section{Method}

- Retrospective cohort study.

- All inborn ELBW infants admitted into a Level 3 NICU from January 2010-December 2013.

- Maternal swabs; mode of delivery; infants SSC, blood cultures and mean CRP (within $72 \mathrm{~h}$ ) were reviewed.

Result

- 161 ELBW infants were admitted and all had admission SSC, CRPs and blood cultures.

- 25 of $161(15.5 \%)$ had positive SSC (Figure 1) of which 5 were mixed culture results.

- 11 of $161(6.8 \%)$ had EONS (positive blood cultures) (Table $1)$.

- 4 of $25(16 \%)$ of positive SSC had correlating blood culture all of which were E coli; 1 subject had positive SSC and blood culture but did not correlate.

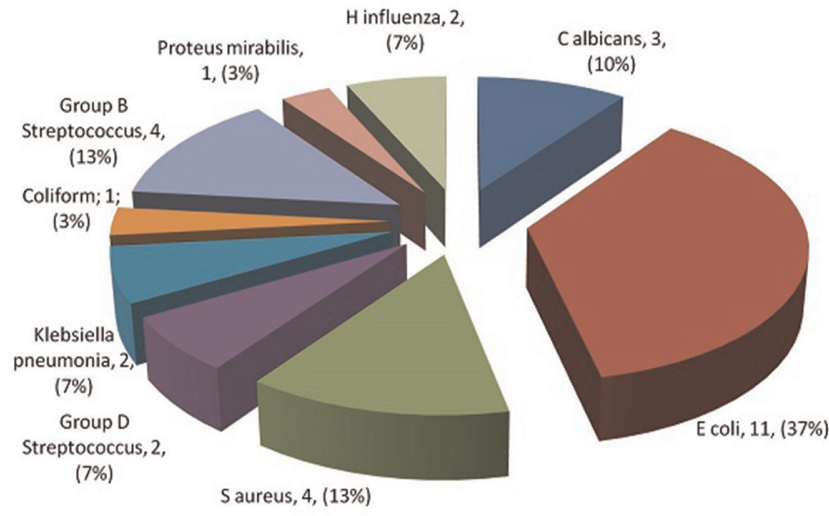

Abstract P0-0555 Figure 1 Bacteria grown from skinj swab

\begin{tabular}{ll}
$\begin{array}{l}\text { Abstract PO-0555 Table 1 } \\
\text { cultures }\end{array}$ & Bacteria grown from initial blood \\
\hline Microorganism & $\mathrm{n} \mathrm{( \% )}$ \\
E coli & $4(36.3)$ \\
S aureus & $1(9.1)$ \\
Gram positive cocci & $1(9.1)$ \\
Group B streptococcus & $1(9.1)$ \\
Peptostreptococcus asaccharolyticus & $1(9.1)$ \\
Coagulase negative staphyloccus & $3(27.2)$ \\
\hline
\end{tabular}

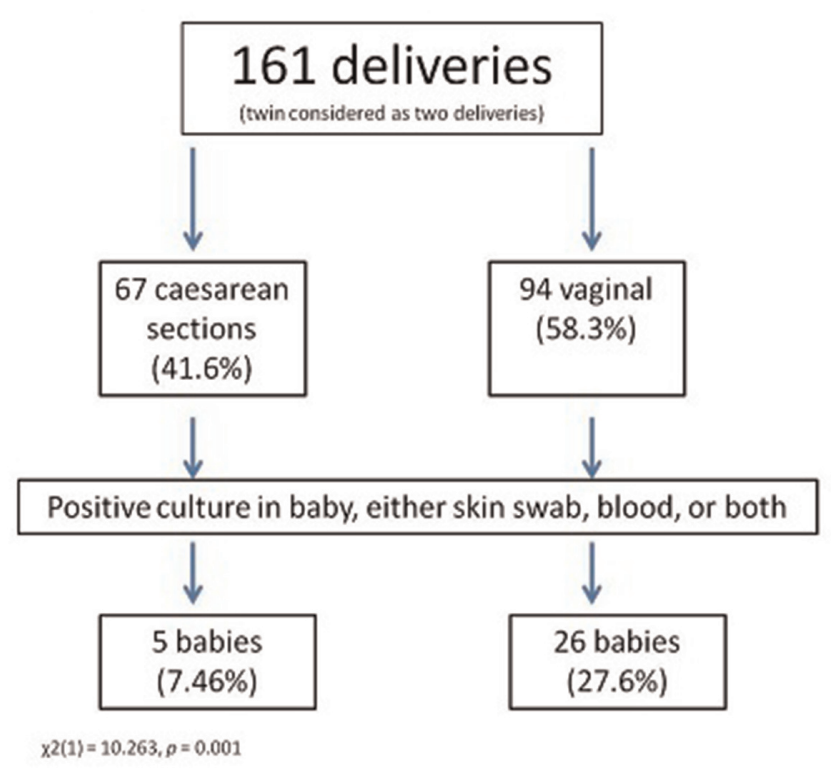

Abstract P0-0555 Figure 2 Percentage of positive microbiological result for different mode of delivery

\begin{tabular}{ll} 
Abstract PO-0555 Table 2 & Mean CRP for different \\
microbiological result & \\
\hline Microbiological result & Mean CRP \\
Skin negative;Blood negative & 5.8 \\
Skin negative;Blood positive & 9.3 \\
Skin positive;Blood negative & 17.2 \\
Skin positive;Blood positive & 15.0 \\
\hline F(3,10.164)=2.026, $\mathrm{P}=0.173$ &
\end{tabular}


- There was a statistically significant association between mode of delivery and positive culture result, $\chi 2(1)=10.263, \mathrm{p}=$ 0.001 (Figure 2).

- Sensitivity, specificity and positive predictive value for skin swab were $36.3 \%, 86.7 \%$ and $16 \%$ respectively.

Conclusions

- Routine SSC is inefficient in predicting the pathogen responsible for sepsis among premature neonates.

- E coli was the predominant organism in the study and $37 \%$ of babies with positive SSC had E. coli sepsis (blood culture).

- Mean CRP was higher in positive skin \pm blood culture cases. However this was statistically not significant.

- There was increased risk of EONS with Vaginal delivery.

\section{PO-0556 HAND HYGIENE IN AN INDIAN NICU: SCOPE FOR IMPROVEMENT}

${ }^{1} \mathrm{R}$ Shah, ${ }^{1} \mathrm{D}$ Patel, ${ }^{1} \mathrm{~S}$ Nimbalkar, ${ }^{1} \mathrm{~K}$ Shah, ${ }^{2} \mathrm{AG}$ Phatak. ${ }^{1}$ Department of Pediatrics, Pramukhswami Medical College, Karamsad, India; ${ }^{2}$ Central Research Services, Charutar Arogya Mandal, Karamsad, India

\subsection{6/archdischild-2014-307384.1198}

Background Stringent handwashing practice is an essential component of patient care in neonatal intensive care units. We evaluated handwashing practices followed in the Neonatal Intensive Care Unit at Shree Krishna Hospital in Karamsad, India.

Methods Prospective observational study over a week in November 2013. Motion activated camera place over washbasin recorded handwashing. Six main steps i.e. step 2 to step 7 of World Health Organisation's hand hygiene technique with soap and water were used for evaluation. Handwashing was categorised as excellent if it exceeded 20 seconds and all six steps were followed, acceptable if duration exceeded 20 seconds but only 3 steps were followed. Rest was classified as unacceptable.

Results Of 1081 recordings, 775 (71.7\%) were nurses, 204 (18.9\%) were parents and $102(9.4 \%)$ were of doctors. From these, 403 (37.3\%) were excellent, 521 (48.2\%) were acceptable and $157(14.5 \%)$ were unacceptable handwash. From nurses, 82 $(10.6 \%)$, from parents $70(34.3 \%)$ and from doctors $5(4.9 \%)$ were unacceptable handwash. There were 665 (61.5\%) occurring during day time (8 am to $8 \mathrm{pm}$ ) and 416 (38.5\%) during nights. Unacceptable handwashing was more prevalent in the night as compared to daytime $(73[17.5 \%])$ vs. 84 [12.6\%], $\mathrm{p}=0.025)$. Twelve people washed their face after washing their hands in the night shift.

Conclusion The study indicates scope for improvement with emphasis on night shifts and parents. Innovative interventions may be required for parents. Empowerment of all stakeholders with reemphasis with constructive feedback may be considered along with repeat cross sectional studies to improve quality.

\section{PO-0557 NEURO-IMAGING IN INFANTS WITH CONGENITAL CYTOMEGALOVIRUS INFECTION: RELATION WITH TIME OF ONSET OF INFECTION DURING PREGNANCY}

${ }^{1} \mathrm{~N}$ Oosterom, ${ }^{1} \mathrm{~J}$ Nijman, ${ }^{1} \mathrm{~J}$ Gunkel, ${ }^{2} \mathrm{TFW}$ Wolfs, ${ }^{1} \mathrm{~F}$ Groenendaal, ${ }^{1} \mathrm{MA}$ Verboon-Maciolek, ${ }^{1} \mathrm{LS}$ de Vries. ${ }^{1}$ Department of Neonatology, Wilhelmina Children's Hospital University Medical Center Utrecht, Utrecht, Netherlands, ${ }^{2}$ Department of Pediatrics, Wilhelmina Children's Hospital University Medical Center Utrecht, Utrecht, Netherlands
Background Cytomegalovirus infection early in pregnancy results in major disabilities, including cerebral palsy and sensorineural hearing loss (SNHL). Cerebral abnormalities detected using cranial ultrasound (cUS) and magnetic resonance imaging (MRI) have been related to neurological sequelae.

Objective To evaluate additional value of MRI and assess relationship between time of infection during pregnancy and outcome in infants with congenital cytomegalovirus (cCMV) infection.

Patients and methods Demographic and clinical data were collected in infants with cCMV infection (1992-2013). Time of onset of infection during pregnancy, neuro-imaging results and outcome were reviewed. Cerebral abnormalities were categorised into none, mild (lenticulostriate vasculopathy (LSV), germinolytic cyst, high signal intensity T2 weighted images) and severe (migrational disorder, ventriculomegaly, cerebellar hypoplasia). Fisher exact test was used for statistical analysis.

Results Thirty-five infants were eligible for analysis. cUS was performed in all and MRI in 19 infants. cUS was superior for diagnosing LSV $(p<0.01)$ and MRI for diagnosing migrational disorders $(\mathrm{p}<0.01)$.

In 17 infants time of onset of infection during pregnancy was ascertained. Eight of ten infants infected during first trimester had severe cerebral abnormalities and adverse sequelae, two had no or mild cerebral abnormalities and normal outcome. Two of three infants infected during second trimester had normal outcome and one developed SNHL. All four infants infected during third trimester had normal outcome.

Conclusion Infants with first trimester cCMV infection are most at risk of severe cerebral abnormalities and neurological sequelae. MRI provides additional information for presence of migrational disorders, essential for early prediction of outcome.

\section{PO-0558 ANTI-INFLUENZA IGG LEVELS IN NEWBORN INFANTS, BORN FROM MOTHERS, VACCINATED FROM INFLUENZA AT PREGNANCY}

${ }^{1} \mathrm{MP}$ Kostinov, ${ }^{2} \mathrm{AP}$ Tcherdantsev, ${ }^{1} \mathrm{D}$ Pakhomov. 'Laboratory of Vaccination and Immunotherapy of Allergic Diseases, Institute of Sera and Vaccines, Moscow, Russia; ${ }^{2}$ Cathedra of Pediatrics, Ulianovsk University, Ulianovsk, Russia

\subsection{6/archdischild-2014-307384.1200}

Background and aims Influenza vaccination with inactivated vaccines is safe for pregnant women, as for fetus. But the levels of immunity protection against influenza in newborn infants, born from mothers, vaccinated by immunoadjuvant vaccines in pregnancy are not investigate yet. We study transplacental IgG levels in newborn infants, born from mothers, vaccinated in pregnancy by subunit influenza vaccines.

Materials and methods We study 79 infants, born from mothers, vaccinated in second and third trimester of normal pregnancy. 42 of them (first group), who's mothers were vaccinated by three-valent subunit vaccine (Grippol Plus by 'Petrovax, Ltd company, Russia, contain 0,5 mcg influenza strains and $500 \mathrm{mcg}$ of Polyoxidonium, 37 (second group) - were from mothers, vaccinated by three valent subunit vaccine ('Agrippal S1' by 'Novartis Vaccines and Diagnostic', Italy) see Table 1. Criteria of Committee for Proprietary Medicinal Products by CPMP/BWP/ 214/96 protocol be used as immunological criteria. IgG-antibody levels were measured in umbilical cord blood by hemagglutination-inhibition reaction.

Results Comparative characteristic of laboratory data achieve that in newborn infants $53,1 \%, 67,6 \%$ and $62,5 \%, 57,7 \%$ were 